\title{
Genome research in pre-dementia stages of Alzheimer's disease
}

\author{
SONIA MORENO-GRAU, AGUSTÍN RUIZ* \\ Memory Clinic of Fundació ACE, Institut Català de Neurociències Aplicades, Barcelona, Spain
}

\begin{abstract}
Genetic characterization of individuals at risk of Alzheimer's disease (AD), i.e. people having amyloid deposits in the brain without symptoms, people suffering from subjective cognitive decline (SCD) or mild cognitive impairment (MCI), has spurred the interests of researchers. However, their pre-dementia genetic profile remains mostly unexplored. In this study, we reviewed the loci related to phenotypes of AD, MCI and SCD from literature and performed the first meta-analyses evaluating the role of apolipoprotein $\mathrm{E}(A P O E)$ in the risk of conversion from a healthy status to MCI and SCD. For AD dementia risk, an increased number of loci have been identified; to date, 28 genes have been associated with Late Onset AD. In MCI syndrome, $A P O E$ is confirmed as a pheno-conversion factor leading from $\mathrm{MCI}$ to $\mathrm{AD}$, and clusterin is a promising candidate. Additionally, our meta-analyses revealed $A P O E$ as genetic risk factor to convert from a healthy status to MCI $\left[\mathrm{OR}=1.849(1.587-2.153) ; P=2.80 \times 10^{-15}\right]$ and to a lesser extent from healthy status to $\mathrm{SCD}[\mathrm{OR}=1.151$ $(1.015-1.304) ; P=0.028]$. Thus, we believe that genetic studies in longitudinal SCD and MCI series may provide new therapeutic targets and improve the existing knowledge of AD. This type of studies must be completed on healthy subjects to better understand the natural disease resistance to brain insults and neurodegeneration.
\end{abstract}

\section{Introduction}

Although Alzheimer's disease (AD) is mainly diagnosed in the elderly, its pathophysiological processes begin several years prior to the onset of symptoms (Ref. 1).

Clinical AD is preceded by a long asymptomatic period, which has been divided into three stages: (1) an initial preclinical stage, (2) a second mild, but progressive, cognitive impairment (MCI) and (3) the final stage of clinical dementia due to $\operatorname{AD}(\operatorname{Refs} 1,2$, $3)$. Recently, researchers have increasingly focused on the characterisation of stages of AD risk, as these provide a critical opportunity for potential intervention (Ref. 1).

With ageing, there is a natural decline in cognitive skills. Thus, it may be difficult to discriminate between early cognitive changes due to $\mathrm{AD}$ and normal ageing process (Ref. 4). In that context, the first evidence of dementia may be the subjective cognitive decline (SCD), defined as a self-reported memory impairment with normal cognitive performance (Ref. 5). Complainers present a higher rate of conversion of SCD to either MCI or dementia (Ref. 6). Thus, epidemiological studies pointed SCD as a predictor of cognitive decline (Refs 7, 8) and as an independent risk factor for dementia (Ref. 9). In succession, the prodromal stage of dementia, MCI, has been defined as memory impairment beyond that expected for normal ageing (Ref. 10). Several MCI phenotypes have been associated with $\mathrm{AD}$ progression
(Ref. 11); however, amnestic MCI (aMCI) confers a higher risk of conversion (Ref. 11).

The identification of MCI subjects, or even SCD who will convert to MCI or dementia, puts across an interesting strategy for secondary prevention of $\mathrm{AD}$. In that sense, the biomarkers of $\beta$-amyloidosis and tau-mediated neuronal injury are detected in subjects with normal cognition (Ref. 12). However, these biomarkers are not sufficient to produce the clinical symptoms of MCI and dementia or are not specific to $\mathrm{AD}$ either (Ref. 1). Furthermore, these biomarkers are not sensitive to disease progression (Ref. 13). Hence, new approaches are required to improve the differentiation of SCD or MCI converters to AD.

AD's genetics has gained much attention since $A D$ presents a heritability of up 70\% (Ref. 14). Recently, researchers have been striving towards the identification of new AD's genetic risk factors. In that sense, the identification of a genetic risk profile for predementia stages may prove to be a powerful approach to select the candidate subjects to prevent or delay the disease progression during the early preclinical stages. If a fraction of SCD and MCI patients are in the pre-AD stages, the identification of an increased number of AD risk alleles as well as that of additional genetic factors specifically influencing SCD or MCI progression can be expected.

In this work, we reviewed the available information in the literature for genome-wide significant variants associated with $\mathrm{AD}$ and their involvement in 
preclinical, prodromal and dementia stages of $\mathrm{AD}$. Additionally, we provide new meta-analysed data for apolipoprotein $\mathrm{E}(A P O E) \varepsilon 4$ in preclinical and prodromal stages.

\section{Methods: meta-analysis}

Meta-analysis was performed for exploring the role of $A P O E \varepsilon 4$ in: (1) risk of $\mathrm{MCI}$ and (2) risk of SCD.

\section{Dataset selection}

Literature search was conducted in PubMed (http:// www.ncbi.nlm.nih.gov/pubmed/) using the following keywords: (1) for MCI: APOE, genetics, risk, mild cognitive impairment and excluding reviews; and (2) for SCD: APOE, SCD. A total of 301 articles were found for MCI and 32 for SCD.

We selected the studies meeting the following criteria: (1) case/control studies or longitudinal studies where it is possible to distinguish a sub-population of cases and a sub-population of controls; (2) studies that provide a complete definition of the participants; (3) studies that evaluated the $A P O E \& 4$ genotype as a risk factor leading to $\mathrm{MCI}$ or $\mathrm{SCD}$, or provided the numbers of $A P O E \& 4$ genotypes or provided sufficient data to calculate them; and (4) studies that provided an odds ratio (OR) with $95 \%$ confidence interval (CI) as well as the $P$-value or provide sufficient data to calculate them. Finally, of the 301 articles found for MCI, 207 did not follow inclusion criteria, 29 showed sample overlapping and 41 had restricted access. A total of 24 articles and 23668 individuals on MCI were finally included. In the case of SCD, 21 of the 32 articles did not follow the inclusion criteria and 3 showed sample overlapping; finally, a total of 8 articles and 6824 individuals were included in the meta-analysis.

\section{Meta-analysis}

Meta-analysis was conducted using the inverse variant method (fixed-effects model) in Ephisheet Excel application. In the case of heterogeneity, DerSimonian and Liard method (random-effects model) was used. Heterogeneity was considered significant when $I^{2}>$ $50 \%$ and $P<0.05$. Meta-analysis results and forest plots were obtained using OpenMeta.

\section{Dementia stage: genetic risk factors of AD}

$\mathrm{AD}$ is a genetically heterogeneous disorder. From a genetic point of view, two patterns of inheritance have been linked to the genomic loci: the autosomal dominant and the polygenic. Traditionally, these patterns have been associated with early and late onset forms of the disease, respectively. However, based on the family history, AD can be subdivided into autosomal dominant, familial and sporadic (Ref. 15).

\section{Autosomal dominant AD}

The familial autosomal dominant pattern in AD represents $\sim 1 \%$ of all the $\mathrm{AD}$ cases and is found almost exclusively in early onset AD (EOAD) (Ref. 15). It occurs in at least three individuals in two or more generations, with two of the individuals being first-degree relatives of the third (Ref. 15).

Linkage and candidate gene studies in EOAD families led to the identification of disease-causing mutations in $\beta$-amyloid precursor protein $(A P P)$, presenilin 1 (PSEN1) and presenilin 2 (PSEN2) genes (Refs 16, 17, 18). Most frequent mutations are shown in PSEN1 and APP loci, respectively, which present complete penetrance in contrast to PSEN2, which presents 95\% penetrance (Ref. 15). These identifications promoted the formulation of amyloid cascade hypothesis, which is still considered as a possible disease mechanism. Despite that, there are EOAD families with negative screening for APP, PSEN1 and PSEN2 mutations supporting the existence of additional causal genes (Ref. 19). In addition, it is seen that $A P O E \& 4$ genotype, the major genetic risk factor for Late-Onset Alzheimer's disease (LOAD) (Ref. 20), also modifies the risk of EOAD (Ref. 21).

Presently, 262 pathogenic mutations have been identified: 42 in APP, 207 in PSEN1 and 13 in PSEN2 (http://www.molgen.ua.ac.be/ADMutations); no other genes have been associated with an autosomal dominant form of $\mathrm{AD}$.

\section{Familial AD (FAD)}

FAD occurs in more than one individual and, at least, two of the affected individuals are third-degree relatives or closer (Ref. 15). Most of the FAD cases are LOAD, but the presence of early onset FAD may be caused by hidden autosomal-dominant AD mutations (Ref. 15).

\section{Sporadic AD (SAD)}

SAD occurs in isolated cases in families or cases separated by more than three degrees of relationship. SAD represents $75 \%$ of all $\mathrm{AD}$ cases and typically presents a LOAD chart (Ref. 15).

\section{The commonest $A D$ phenotype, $L O A D$}

The genetic and molecular basis for the commonest AD phenotype, i.e. LOAD, remains widely unknown. However, important progress on the isolation of the loci associated with $\mathrm{AD}$ has been achieved in the past few years because of the emergence of the genomewide association (GWAS) and exome studies.

The $\varepsilon 4$ allele of the $A P O E$ gene was the first genetic variant associated with LOAD (Ref. 20), and it remains as the major risk factor for the disease until now.

Behind the $A P O E$ discovery, the candidate gene approach led to the identification of two clusters of single nucleotide polymorphisms (SNPs) in SORL1 gene (Ref. 22). Recently, this association has been validated by International Genomics Alzheimer's Project (IGAP) (Ref. 23). However, candidate gene approach did not show more successful outcomes. 
Most discoveries arrived when the GWAS strategy was applied to the large case-controlled datasets. In the GWAS era, common variants located at $C L U$, PICALM, CR1, BIN1, ABCA7, CD2AP, CD33, EPHA1 and MS4A6A-MS4A4E loci were associated with LOAD (Refs 24, 25, 26).

To validate the original GWAS findings, replication studies were performed with many independent datasets. Consequently, CR1, PICALM, CLU and BIN1 signals have been replicated in Caucasians (Refs 27, 28), Caribbean Hispanics (Ref. 29) or Asian individuals (Refs 30, 31). In addition, the EPHA1 and CD33 genetic variants were replicated in Caucasian subjects (Ref. 32), and $A B C A 7$ in African Americans (Ref. 33).

A recent meta-analysis developed by IGAP in 74 046 individuals of European ancestry confirmed previously reported GWAS signals $(A B C A 7, B I N 1, C L U$, CR1, CD2AP, EPHA1, MS4A6A-MS4A4E and PICALM) (Ref. 23). Nevertheless, the CD33 locus, previously associated with LOAD, did not reach the genome-wide significance in the replication stages (Ref. 23). Moreover, the IGAP meta-analysis identified 10 novel genetic regions associated with LOAD: CASS4, SLC2A4A-RIN1, FERMT2, HLA-DRB5-HLADRB1, INPP5, MEF2C, PTK2B, CELF1, NME8 and $Z C W P W 1$ and also confirmed a candidate gene, SORL1 (Ref. 23). Follow-up studies of the IGAP results revealed additional locus namely TRIP4 (Ref. 34) and a novel AD locus within the microtubule-associated protein tau $(M A P T)$ region at 17q21.31 (Ref. 35). Finally, the gene-wide analyses of the IGAP dataset identified TP53INP1 and IGHV1-67 as the novel AD loci (Ref. 36), and an independent meta-analysis identified ATP5H/KCTD2 as the LOAD risk signal (Ref. 37). Most loci reported after the initial IGAP report would require independent replications confirming its plausibility.

Despite that, the GWAS approach presented a disadvantage, i.e. its inability to detect rare variants, which might be a source of functional variants with larger effects on the LOAD risk (Ref. 38). This lack was covered by the implementation of genome and exome sequencing technologies. Thus, in the recent years, rare variants with a significant effect on the risk for LOAD have been identified in APP, TREM2, PDL3 and $U N C 5 C$ loci (Refs 38, 39, 40, 41). However, more efforts are needed to confirm the original signals. Several studies have confirmed the reported association of TREM2 with LOAD (Refs 42, 43). In addition, the existence of TREM2 variants associated with the Naso-Hakola disease (Ref. 44) and frontotemporal dementia (Ref. 45) supports its role in neurodegeneration. Alternatively, the PLD3 variants' replication did not replicate the previous effect or overall burden analyses (Refs 46, 47). Therefore, prudence is required to define the genuine signals associated with rare variants.

At present, 28 genetic regions have been associated with LOAD (Table 1), but many of them still require independent validation. These genes can be divided into four major functional clusters: (i) amyloid beta (A $\beta$ ) metabolism (APOE, CLU, ABCA7, CASS4, SORL1 and APP), (ii) Tau metabolism (BIN1, SLC2A4A-RIN1, CASS4, FERMT2 and 17q21.31 $M A P T$ region), (iii) synaptic function (PICALM, CD2AP, EPHA1, SLC2A4A-RINI, MEF $2 C$ and $Z C W P W 1$ ) and (iv) immune response and inflammation (CLU, CR1, EPHA1, MS4A cluster, ABCA7, HLA-DRB5-HLA-DRB1, INPP5; MEF2C, TREM2 and IGHV1-67). Seven identified loci do not have a well-established pathway (PTK2B, CELF1, NME8, TRIP4, ATP5H/KCTD2, UNC5C and TP53INP1) (Fig. 1).

Along with the identification of single locus, GWAS also permits the genetic confirmation of candidate pathways. Recently, pathway analysis studies have pointed toward the crucial role of the immune system in $\mathrm{AD}$ (Ref. 48), that has been further reinforced by the IGAP results (Ref. 49). Moreover, the IGAP study also implicates the regulation of endocytosis, cholesterol transport and protein ubiquitination as prime targets in the aetiology of AD (Ref. 49). The knowledge of the biological pathways involved in disease aetiology is crucial in the development of therapeutic strategies to aid in the prevention or treatment of LOAD.

\section{Prodromal stage: genetics of mild cognitive impairment syndrome}

\section{APOE genotype in MCI}

Petersen et al. (Ref. 50) were the first to provide evidence that MCI subjects with at least one allele of $A P O E \& 4$ presented a higher probability of conversion to dementia. Although subsequent genetic studies supported it (Refs 51, 52), they had small sample sizes, which only succeeded in providing an approximate value of the risk effect (Ref. 53). Thus, the metaanalysis conducted by Elias-Sonnenschein et al. (Ref. 54) provided the first consistent data corroborating the role of $A P O E \varepsilon 4$ as a genetic risk factor for progression from MCI to $\mathrm{AD}$ (Table 2).

The involvement of $A P O E \& 4$ as a risk factor for MCI remains less explored. Therefore, here we have explored the risk conferred by $A P O E$ \&4 genotype to suffer MCI. Our meta-analysis, which includes 23 668 individuals of different ethnic groups, confirmed a significant risk association of $A P O E$ \& 4 genotype and $\mathrm{MCI} \quad[\mathrm{OR}=1.849 \quad(1.587-2.153)] \quad($ Fig. 2) (Table 2). Our dataset showed high heterogeneity $\left(I^{2}=63 \% ; P\right.$-value $\left.<0.001\right)$. In that sense, sub-population study revealed higher heterogeneity for Caucasian dataset with respect to Asiatic group $\left(I^{2}=\right.$ $68 \% ; P$-value $<0.001 ; I^{2}=51 \%, P$-value $\left.=0.104\right)$. It must be considered that the major number of available studies is provided for Caucasians. Additionally, several studies have shown that the risk is higher for aMCI subpopulation in comparison with the rest of 
TABLE 1

GENETIC REGIONS ASSOCIATED WITH LOAD FROM HIGHEST TO LOWEST ODDS RATIO.

\begin{tabular}{|c|c|c|c|c|}
\hline Gene & Marker & OR $(95 \% \mathrm{CI})$ & $P$-value & Author \\
\hline \multicolumn{5}{|l|}{ Common variants } \\
\hline$A P O E$ & rs429358 & $3.68(3.30-4.11)$ & $9.3 \times 10^{-120}$ & Corder et al. $(20)^{\mathrm{b}}$ \\
\hline ATP5H/KCTD2 & rs11870474 & $1.53(1.33-1.77)$ & $4.7 \times 10^{-9}$ & Boada et al. (37) \\
\hline TRIP4 & rs74615166 & $1.31(1.17-1.42)$ & $9.74 \times 10^{-9}$ & Ruiz et al. (34) \\
\hline BIN1 & rs6733839 & $1.22(1.18-1.25)$ & $6.9 \times 10^{-44}$ & Seshadri et al. $(25)^{\mathrm{c}}$ \\
\hline$A B C A 7$ & rs4147929 & $1.15(1.11-1.19)$ & $1.1 \times 10^{-15}$ & Hollingworth et al. (26) \\
\hline CR1 & rs6656401 & $1.18(1.14-1.22)$ & $5.7 \times 10^{-24}$ & Lambert et al. (119) \\
\hline FERMT2 & rs17125944 & $1.14(1.09-1.19)$ & $7.9 \times 10^{-9}$ & Lambert et al. (23) \\
\hline$H L A-D R B 5$ /DRB1 & rs9271192 & $1.11(1.08-1.15)$ & $2.9 \times 10^{-12}$ & Lambert et al. (23) \\
\hline$P T K 2 B$ & rs28834970 & $1.10(1.08-1.13)$ & $7.4 \times 10^{-14}$ & Lambert et al. (23) \\
\hline$C D 2 A P$ & rs10948363 & $1.10(1.07-1.13)$ & $5.2 \times 10^{-11}$ & Hollingworth et al. (26) \\
\hline INPP5D & rs35349669 & $1.08(1.05-1.11)$ & $3.2 \times 10^{-8}$ & Lambert et al. (23) \\
\hline$C E L F 1$ & rs 10838725 & $1.08(1.05-1.11)$ & $1.1 \times 10^{-8}$ & Lambert et al. (23) \\
\hline NME8 & rs2718058 & $0.93(0.90-0.95)$ & $4.8 \times 10^{-9}$ & Lambert et al. (23) \\
\hline$M E F 2 C$ & rs 190982 & $0.93(0.90-0.95)$ & $3.2 \times 10^{-8}$ & Lambert et al. (23) \\
\hline$Z C W P W 1$ & rs 1476679 & $0.91(0.89-0.94)$ & $5.6 \times 10^{-10}$ & Lambert et al. (23) \\
\hline$S L C 24 A 4-R I N 3$ & rs 10498633 & $0.91(0.88-0.94)$ & $5.5 \times 10^{-9}$ & Lambert et al. (23) \\
\hline$C D 33^{\mathrm{a}}$ & rs3865444 & $0.91(0.88-0.93)$ & $1.6 \times 10^{-9}$ & Hollingworth et al. (26) \\
\hline$M S 4 A$ & rs983392 & $0.90(0.87-0.92)$ & $6.1 \times 10^{-16}$ & Hollingworth et al. (26) \\
\hline EPHA1 & rs11771145 & $0.90(0.88-0.93)$ & $1.1 \times 10^{-13}$ & Hollingworth et al. (26) \\
\hline CASS4 & rs7274581 & $0.88(0.84-0.92)$ & $2.5 \times 10^{-8}$ & Lambert et al. (23) \\
\hline PICALM & rs10792832 & $0.87(0.85-0.89)$ & $9.3 \times 10^{-26}$ & Harold et al. $(24)^{\mathrm{c}}$ \\
\hline$C L U$ & rs9331896 & $0.86(0.84-0.89)$ & $2.8 \times 10^{-25}$ & Harold et al. $(24)^{c}$ \\
\hline SORL1 & rs1 1218343 & $0.77(0.72-0.82)$ & $9.7 \times 10^{-15}$ & Lambert et al. (23) \\
\hline IGHVI-67 & NA & NA & $7.9 \times 10^{-8}$ & Escott-Price (36) \\
\hline TP53INP1 & NA & NA & $1.4 \times 10^{-6}$ & Escott-Price (36) \\
\hline $17 \mathrm{q} 21.31$ region & rs 2732703 & $0.73(0.65-0.81)$ & $5.8 \times 10^{-9}$ & Jun et al. (35) \\
\hline$A P O E$ & rs7412 & $0.62(0.46-0.85)$ & $2.7 \times 10^{-3}$ & Corder et al. $(20)^{\mathrm{b}}$ \\
\hline \multicolumn{5}{|l|}{ Rare variants } \\
\hline TREM2 & rs75932628 & $5.05(2.77-9.16)$ & $9.0 \times 10^{-9}$ & Guerreiro et al. (34) \\
\hline UNC5C & rs 137875858 & $2.15(1.21-3.84)$ & $9.5 \times 10^{-3}$ & Wetzel-Smith et al. (45) \\
\hline$P L D 3^{\mathrm{a}}$ & rs145999145 & $2.10(1.47-2.99)$ & $2.9 \times 10^{-5}$ & Cruchaga et al. (40) \\
\hline$A P P$ & rs63750847 & 0.24 (NA) & $4.2 \times 10^{-5}$ & Jonsson et al. (38) \\
\hline
\end{tabular}

${ }^{a}$ Not replicated in follow-up studies or IGAP. ${ }^{b}$ Estimator extracted from Bertram et al. (118). ${ }^{c}$ Estimator extracted from Lambert et al. (23). IGAP: International Genomics Alzheimer's Project; LOAD: late onset Alzheimer's disease; NA: not available; OR: odds ratio.

the MCI subtypes (Ref. 55) (Table 2). This makes sense in the context of MCI as the prodromal stage of AD.

Although most of the attention has been focused on the risk allele $\varepsilon 4$ of $A P O E$, the $\varepsilon 2$ allele has also demonstrated its role in AD (Ref. 56). Some studies have shown that cognitively normal carriers of $\varepsilon 2$ allele were less likely to present with cognitive decline $($ Refs 57,58$)$ and develop $\mathrm{AD}$ [relative risk $(\mathrm{RR})=$ $0.76(0.40-1.44)]$ (Ref. 57). Others showed that the MCI patients with $\varepsilon 2$ allele had a better memory in comparison with non-carriers (Ref. 59). Similarly, a recent study also detected the protective effect of $A P O E$ \&2 against pheno-conversion from MCI to $\mathrm{AD}$ $[\mathrm{HR}=0.69$ (0.51-0.86), $P=0.004]$ (Ref. 60). These observations suggest the role of $\varepsilon 2$ allele in the protection against LOAD and its importance as a possible mechanism to reverse the APOE $\varepsilon 4$ effect (Ref. 60).

\section{Non-APOE LOAD loci in MCI}

Apart from $A P O E$, the genome-wide significant variants for LOAD in MCI population remain largely unknown. However, in the past few years, several studies have pointed out their influence in cognitive decline. Thus, $C R 1$ and $A B C A 7$ genes have been associated with faster rate of cognitive decline (CRI
$P$-value $=0.011 ; A B C A 7 P$-value $=0.013)(\operatorname{Refs} 61$, 62). EPHAI and PICALM loci were also associated with faster and slower rate of decline (EPHA1 P= 0.013; PICALM $P=0.027$ ), respectively, however they did not support Bonferroni corrections (Ref. 63). Following studies for EPHA1 only replicated it marginally $(P=0.05)$ (Ref. 62). Finally, the $C L U$ locus was associated with cognitive endophenotypes in several studies. In that sense, the $C L U$ risk allele has been associated with a faster rate of decline in some neuropsychological characteristics such as verbal immediate $(P=0.0032)$ and delayed free recall $(P=0.032)$ (Ref. 64) and its protective allele with a decreased risk of conversion to $\mathrm{AD}[\mathrm{OR}=0.25(0.07-0.84)$, $P=0.025]$ (Ref. 65). A study that evaluated the progression of normal subjects $(n>2000)$ to MCI/ LOAD pointed the significant effect of $C L U$ on logical memory delayed recognition (LMDR) (coefficient for $\mathrm{LMDR}=-0.51 \quad(-0.92$ to $-0.11, P=$ 0.012) (Ref. 62). It further showed a borderline significant hazard ratio (HR) in the sensitivity analysis between $C L U$ risk allele and the risk of progression from MCI to $\mathrm{AD}(\mathrm{HR}=1.10, P=0.13$; sensitivity $\mathrm{HZ}=1.14, P=0.049$ ) (Ref. 62). Recently, another experiment conducted on $3326 \mathrm{MCI}$ subjects of four 


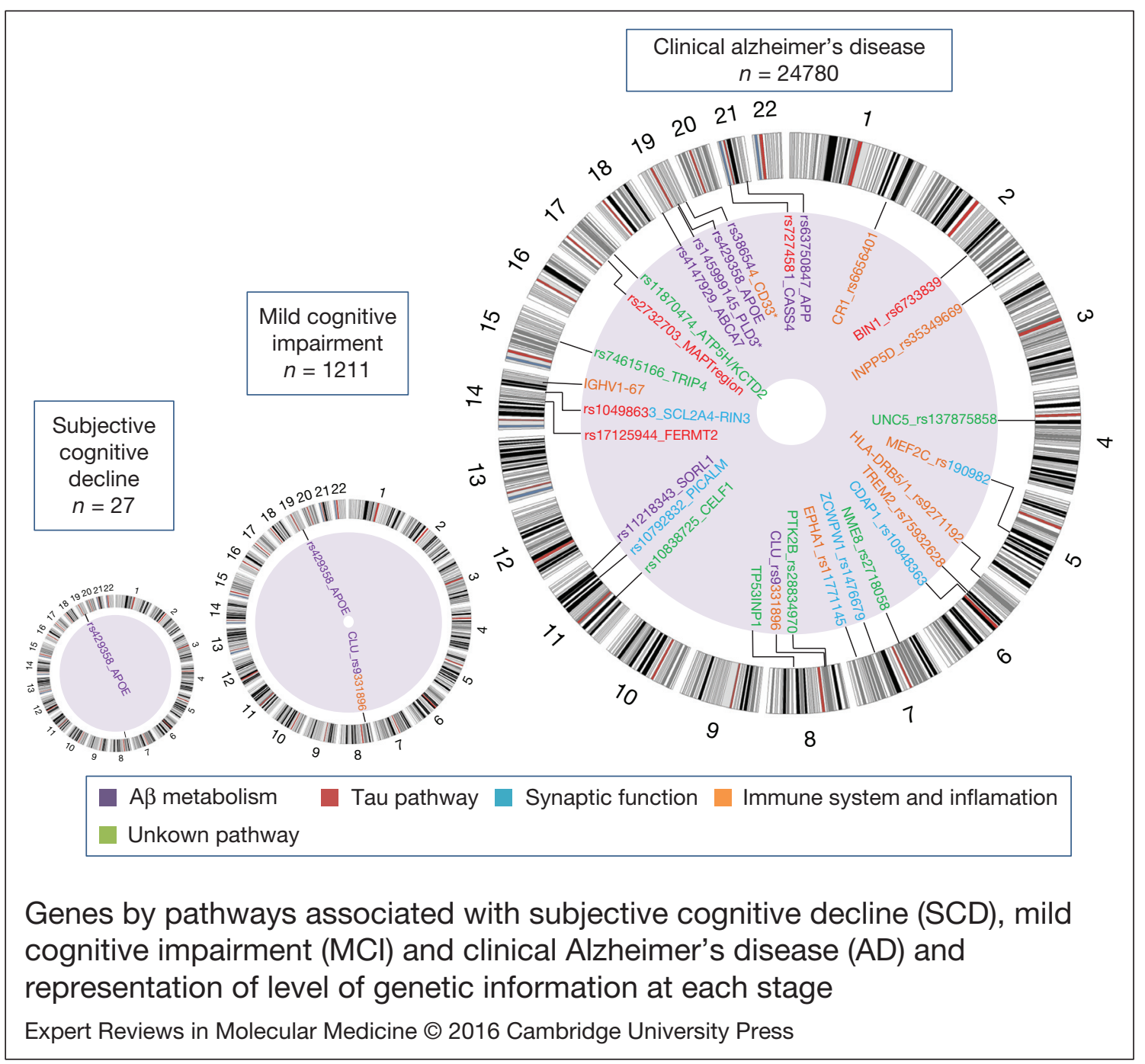

FIGURE 1

Genes by pathways associated with subjective cognitive decline (SCD), mild cognitive impairment (MCI) and clinical Alzheimer's disease (AD) and representation of level of genetic information at each stage. Genetic information response to the number of articles $(n)$ found in the PubMed database with the keywords: Alzheimer's disease and genetics; mild cognitive impairment and genetics; subjective cognitive decline and genetics. Circular ideogram was performed using Circos (Ref. 120). *Not replicated in follow-up studies or in International Genomics Alzheimer's Project (IGAP).

TABLE 2.

GENETIC VARIANTS ASSOCIATED WITH RISK OR PROGRESSION TO DEMENTIA IN MCI AND SCD SUBJECTS.

\begin{tabular}{|c|c|c|c|c|c|}
\hline & Gene & Marker & OR $(95 \%, C I)$ & $P$-value & Type of study \\
\hline \multicolumn{6}{|c|}{ Mild cognitive impairment } \\
\hline \multirow[t]{2}{*}{ Risk factors } & $A P O E$ & rs429358 & $1.85(1.59-2.15)$ & $2.8 \times 10^{-15}$ & Meta-analysis \\
\hline & & & $2.50(1.13-5.69)^{\mathrm{a}}$ & 0.020 & Longitudinal study (55) \\
\hline \multirow[t]{8}{*}{ Progression factors } & $A P O E$ & rs429358 & $2.29(1.88-2.8)$ & $<0.001$ & Meta-analysis (54) \\
\hline & $C L U$ & rs11136000 & $1.19(0.05-1.32)^{\mathrm{b}}$ & 0.003 & GWAS (60) \\
\hline & ACCOT11 & rs1275288 & $2.26(1.56-3.26)^{\mathrm{b}}$ & $<0.001$ & GWAS (63) \\
\hline & UBR5-RRM2B & rs 7840202 & $1.75(1.21-2.64)^{\mathrm{b}}$ & $<0.001$ & GWAS (63) \\
\hline & Unknown & rs11637611 & $2.15(1.4-3.31)^{\mathrm{b}}$ & $<0.001$ & GWAS (63) \\
\hline & $M A P T$ & H1 haplotype & $2.31(1.52-3.51)$ & 0.001 & Candidate Gene (68) \\
\hline & 5-HTT & $\mathrm{S}$ allele & $1.73(1.05-2.84)$ & 0.002 & Candidate Gene (74) \\
\hline & $A D R A P 2 B$ & Deletion (301-303) & $0.49(0.27-0.89)$ & 0.021 & Candidate Gene (76) \\
\hline \multicolumn{6}{|c|}{ Subjective cognitive decline } \\
\hline Risk factor & $A P O E$ & rs429358 & $1.15(1.02-1.30)^{\mathrm{b}}$ & 0.028 & Meta-analysis \\
\hline
\end{tabular}

${ }^{\mathrm{a} A m n e s t i c ~ M C I .}$

${ }^{\mathrm{b}}$ Parameter of association measure hazard ratio; MCI: mild cognitive impairment; SCD: subjective cognitive decline; OR: odds ratio. 


\begin{tabular}{|c|c|c|c|c|c|c|c|c|}
\hline \multicolumn{5}{|l|}{${ }^{\text {a }}$ Risk to $\mathrm{MCl}$} & \multicolumn{4}{|l|}{${ }^{\text {b }}$ Risk to SCD } \\
\hline Study & $\mathbf{N}$ & OR (95\% C.I.) & & & & & & \\
\hline China,P(Ref.121) & 131 & $4.364(1.903,10.010)$ & & & & & & \\
\hline China,W (Ref.122) & 246 & $2.010(0.992,4.447)$ & & & & & & \\
\hline $\begin{array}{l}\text { Japan (Ref. 123) } \\
\end{array}$ & 1,433 & $1.534(1.125,2.091)$ & & $\rightarrow$ & & & & \\
\hline s. Korea (Ref. 124) & 50 & $1.000(0.239,4.184)$ & & & & & & \\
\hline Australia (Ref.125) & 895 & $2.605(1.785,3.802)$ & & & & & & \\
\hline Canada (Ref.126) & 45 & $2.500(0.724,8,635)$ & & & & & & \\
\hline France (Ref.127) & 96 & $6.012(2.237,16.016)$ & & & & & & \\
\hline Germany (Ref. 128) & 2,331 & $1.370(1.020,1.840)$ & & 1 & & & & \\
\hline Greece (Ref.76) & 192 & $1.445(0.784,2.662)$ & & & & & & \\
\hline Italy, B (Ref.71) & 243 & $1.773(0.999,3.148)$ & & & & & & \\
\hline Italy, $\mathbf{P}$ (Ref. 129) & 97 & $1.702(0.625,4.634)$ & & 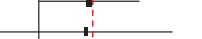 & & & & \\
\hline$\overline{\text { Poland (Ref.130) }}$ & 99 & $2.883(1,172,6.848)$ & & 1, & & & & \\
\hline$\overline{\text { Spain,C (Ref. 131) }}$ & 185 & $1.165(0.766,3.446)$ & & 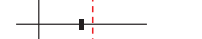 & & & & \\
\hline Spain,P(Ref.1 132) & 1,309 & $3.584(2.764,4.648)$ & & 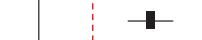 & & & & \\
\hline Sweden (Ref. 133) & 60 & $3.187(1.084,9.373)$ & & $\begin{array}{c:c}1 & 1 \\
& 1\end{array}$ & & & & \\
\hline Switzerland (Ref. 134) & 252 & $1.432(0.602,3.167)$ & & $1:$ & Study & $\mathbf{N}$ & OR (95\% C.I.) & \\
\hline USA,CHS (Ret.55) & 2,895 & $1.900(1.140,1.490)$ & & -1 & Afri-Carib (Ref.140) & 166 & $2.200(0.900,5.378)$ & 1 \\
\hline USA,FOS (Ref. 135) & 2,239 & $1.020(0.6981 .370)$ & & $\longrightarrow$ & China (Ref.121) & 17 & $2.420(0.628,9.330)$ & \\
\hline USA,NAC (Ref. 136) & 8,084 & $1.860(1.688,2.050)$ & & & Australia (Ref. 141) & 301 & $0.780(0.417,1.460)$ & ! \\
\hline USA, NS (Ref. 137) & 470 & $1.870(1.200,2.753)$ & & 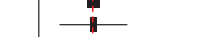 & $\overline{\text { Australia, OPH (Ref.142) }}$ & 147 & $2.350(1.1110,4.975)$ & \\
\hline USA, TF (Ref. 138) & 993 & $1.550(1.142,2.104)$ & & $\rightarrow$ & $\overline{G e r m a n y ~(R e f .143) ~}$ & 2,011 & $1.020(0.810,1.284)$ & + \\
\hline USA,ROS (Ref.139) & 607 & $1.500(1.020,2.206)$ & & & USA,ADNI (Ref.144) & 289 & $1.150(0.660,2,004)$ & \\
\hline Hispanics (Ref.138) & 615 & $1.280(0.836,1.960)$ & & 1 & USA, APOE (Ref.145) & 447 & $\overline{0.800(0.520,1.231)}$ & \\
\hline Brazil (Ref. 72) & 101 & $1.800(0.900,3.600)$ & & i & USA, NHS (Ref.146) & 3375 & $1.260(1.050,1.512)$ & 1 \\
\hline \multirow[t]{2}{*}{$\begin{array}{l}\text { Overall } \\
1=63 \% \\
p<0,001\end{array}$} & 23,668 & $\begin{array}{l}1.849(1.587,2.153) \\
P_{M}=2.80 \times 10^{-15}\end{array}$ & & $\diamond$ & $\begin{array}{l}\text { Overall } \\
R^{2}=46 \%, \\
P=0,775\end{array}$ & 6,824 & $\begin{array}{l}1.151(1.015,1.304) \\
\mathrm{P}_{M}=0.028\end{array}$ & $\Leftrightarrow$ \\
\hline & & 0.24 & 0.48 & $\begin{array}{l}1.21 .85 .78 \\
\text { Odds ratio (log scale) }\end{array}$ & 16.16 & & $\begin{array}{rr}0.42 & 0.831 \\
\text { Od }\end{array}$ & $\begin{array}{llll}31.15 & 2.08 & 4.17 & 8.33 \\
\text { Odds ratio (log scale) }\end{array}$ \\
\hline \multicolumn{9}{|c|}{ Forest plot for APOE \&4 genotype in a) risk to $\mathrm{MCl}$ and b) risk to SCD } \\
\hline \multicolumn{9}{|c|}{ Expert Reviews in Molecular Medicine (C 2016 Cambridge University Press } \\
\hline
\end{tabular}

FIGURE 2

Forest plot for $A P O E$ \&4 genotype in (a) risk to mild cognitive impairment (MCI) and (b) risk to subjective cognitive decline (SCD). OR: odds ratio.

countries supported the association of the $C L U$ locus with the conversion from $\mathrm{MCI}$ to $\mathrm{AD}[\mathrm{HR}=1.19$ $(0.05-1.32), P=0.0035]$ (Ref. 60) (Table 2).

Most of the identified LOAD loci present small risk effects and are, therefore, not quite informative for risk prediction on their own (Ref. 65). Consequently, it is apprehended that the use of the genetic risk score (GRS) strategy, where multiple loci with modest effects are combined, might improve the identification of people at risk for common diseases (Ref. 65). It was observed that $\mathrm{MCI}$ carriers of six or more non- $A P O E$ LOAD risk alleles showed rapid conversion to $A D$ (Ref. 65). However, in another study, the significant effect was only reached when the $A P O E$ genotype was considered $\left(\mathrm{HR}=1.29, P=1.14 \times 10^{-9}\right.$; sensitivity $\left.\mathrm{HR}=1.32, P=5.73 \times 10^{-10}\right)($ Ref. 62). In a recent study, GRS for 19 LOAD loci with genome-wide significance was associated with $\mathrm{MCI}(\mathrm{OR}=1.15$, $P=0.011)$ and with progression from MCI to dementia $(\mathrm{HR}=1.59, P<0.001)($ Ref. 66).

With the exception of the $A P O E$ and $C L U$ variants, it seems difficult to arrive at a conclusion about the role of LOAD SNPs in the context of MCI. Studies developed in larger cohorts are needed to check and validate the expected association between the LOAD risk genetic variants and $\mathrm{MCI}$.

\section{New loci associated with MCI}

It has been observed that additional loci have been associated with MCI. Recently, a GWAS associated rs12752888 (ACOT11 gene), rs7840202 (UBR5 $R R M 2 B$ region) and rs11637611 (unknown gene) markers with MCI progression (Ref. 63) (Table 2). However, establishing a relationship with the present pathophysiological hypothesis of $\mathrm{AD}$, at this stage, seems complex. Consequently, these signals require validation if they were to be discarded as false positive and either to be accepted as factors responsible for MCI progression.

Alternatively, candidate gene studies have suggested several aspirant genes associated with the pheno-conversion of MCI to $A D$. One such example is the $M A P T$ gene. Specifically, a study revealed that the H1/H1 haplotype carriers presented a higher conversion rate of MCI to dementia (Ref. 67). Moreover, the $\mathrm{H} 1$ haplotype has been associated with the risk of aMCI in converters to $\mathrm{AD}$ and non-converters (Ref. 68) (Table 2). Recently, the region17q21.31, 
where MAPT was found, has been associated with LOAD reaching GWAS significance in non- $A P O E$ carriers (Ref. 35). However, it remains unknown whether the casual variant is located in the $M A P T$ or the nearby genes (Ref. 35). For this reason, the MAPT linkage with MCI was explained at this point. Although MAPT is expected to be associated with LOAD because of the role of Tau protein in the classical AD hallmarks and the existence of several studies pointing towards this relationship (Refs 69, 70), prudence is required until the location of the causal signal is identified.

Other genes associated with the pheno-conversion of $\mathrm{MCI}$ to $\mathrm{AD}$ are the vascular endothelial growth factor (Ref. 71), the brain-derived neurotrophic factor $(B D N F)$ (Ref. 72) or butyrylcholinesterase (BCHE) (Ref. 73). An additional marker in the serotonin transporter (5HTT) gene has also been related to MCI (Ref. 74). It has also been associated with an emotion-induced retrograde amnesia (Ref. 75), highlighting the role of serotonin in the memory system. The last reported association with MCI is detected in the $a 2 b$-adrenergic receptor (ADRAP2B) $[\mathrm{OR}=$ 0.491 (0.268-0.899); $P=0.021]$; this association is also identified in $\mathrm{AD}$ subjects $[\mathrm{OR}=0.463$ (0.261-0.822); $P=0.009$ ] (Ref. 76) (Table 2). Since none of these genes have been validated for LOAD, it can be said that they may act as genetic progression factors. They are capable of modulating the rate of decline but are not involved in the risk leading to $\mathrm{AD}$.

\section{Preclinical stage: SCD}

There is limited research on the genetic variants that determine the risk to SCD or the progression of SCD to $\mathrm{MCI}$ or $\mathrm{AD}$. Therefore, we have conducted the first meta-analysis exploring the involvement of $A P O E \& 4$ in the risk to suffer SCD. A significant risk effect was detected $[\mathrm{OR}=1.151 \quad(1.015-1.304)]$ (Fig. 2) (Table 2), with a borderline non-significant heterogeneity $\left(I^{2}=46 \%, \quad P\right.$-value $\left.=0.075\right)$, which remains when the analysis is only performed for Caucasians $\left(I^{2}=48 \%, P\right.$-value $\left.=0.087\right)$. However, this significant association disappears $[\mathrm{OR}=1.158$ (0.933-1.437); $P=0.184]$ when a random model is used to conduct the meta-analysis. From our point of view, these results must be taken with prudence. SCD individuals represent a mixed population, where a pool of subjects may develop dementia, not exclusively $\mathrm{AD}$ and others never develop it. Hence, the sample size needed to detect Alzheimer's genuine genes must be larger.

Apart from the $A P O E$ \&4 polymorphism, other markers have been investigated to assess their possible association with SCD, such as alpha-2 macroglobulin gene (Ref. 77), presenilin-1 mutation Glu318Gly (Ref. 78), gene polymorphisms involved in vascular alterations (Ref. 79) and inflammatory genes (Ref. 80). However, all these studies did not report any association with SCD.
The genetic profile of the SCD subjects is unexplored in spite of the fact that its analysis could provide new ways to manage the disease. The generation of large SCD datasets integrating genomic information with follow-up data would be an essential step in identifying genetic elements responsible for the progression of SCD to MCI and $\mathrm{AD}$.

\section{Other approaches: endophenotype-based approach}

The use of quantitative traits closely related to the disease state, namely, endophenotypes, has been proposed as a simpler way to deal with genetic testing of LOAD. Thus, several endophenotypes have emerged across the cognitive spectrum of AD.

\section{Differential amyloid burden and brain volume as endophenotype}

Greater amyloid positron emission tomographic (PET) uptake is detected in $\mathrm{AD}, \mathrm{MCI}$ and $\mathrm{SCD}$ cases as well as healthy controls who are carriers of the $\varepsilon 4$ allele of $A P O E$ gene (Refs 81,82$)$. APOE \&4 carriers become positive for amyloid PET imaging earlier (Ref. 81) and show a higher cognitive decline (Ref. 83). Moreover, signals in $A P O E$ locus have been detected by GWAS of longitudinal studies for change in amyloid burden (Ref. 84). Thus, in the past few years, $A P O E$ contribution to the determination of $\mathrm{AD}$ dementia converters has been reinforced. In addition, recent GWAS of longitudinal studies has also provided novel genetic correlations with the amyloid burden, such as $B C H E$, TREMI and ILRIRAP (Refs 83, 85, 86).

Findings around differential brain volumes have also involved LOAD loci showing that a reduced hippocampal volume (HCV) is associated with SORL1 in AD patients (Ref. 87) and CLU gene in young healthy controls (Ref. 88). HLA-DRB1 locus was correlated with a decrease in total brain volume along large longitudinal cohorts (Ref. 89). Putamen volume was also associated with genetic variants, involved in apoptosis, axon guidance and vesicle transport (Ref. 90). In that context, axon guidance pathway was also associated with reduced $\mathrm{HCV}$, as well as calcium and ErbB signalling (Ref. 91). In addition, GRS for LOAD risk variants was associated with cortical thickness (Ref. 92) and reduced $\mathrm{HCV}$ in cognitively normal subjects, although $\mathrm{HCV}$ association disappears after removing $A P O E$ locus (Ref. 89).

\section{Amyloid- $\beta$ and tau levels in cerebrospinal fluid (CSF)}

CSF levels of A $\beta 42$ and pTau181 have also been used as LOAD endophenotypes. APOE locus has been associated with both $A \beta 42$ (Refs 93, 94, 95) and pTau181 levels (Ref. 94). However, Elias-Sonnenschein et al. (Ref. 93) showed the correlations of $A P O E$ with A $\beta 42$ but not with CSF tau biomarkers. Correlations with other LOAD loci remain scarce. Kauwe et al. (Ref. 96) did not find any association for BINI, CRl, $C L U$ and PICALM, although, recently, CLU and 
MSA4A have been associated with A $\beta 42$ levels (Ref. 93). In addition, GRS for AD loci did not provide any association (Ref. 97). Novel identifications have pointed to the $3 \mathrm{q} 28$ region, GLIS3 gene and TREM cluster association with tau biomarkers (Ref. 94) and SUCLG2 association with A $\beta 42$ levels (Ref. 95). For further information, refer to Cruchaga et al. (Ref. 98).

There is an inverse correlation between brain amyloid burden and A $\beta$ CSF levels (Ref. 99). From our understanding, both techniques are dealing with the same pathological process, $A \beta$ deregulation. In that scenario, the identification of the following two might be expected: (1) the same genetic factors independently of the analysed quantitative trait and (2) the reported LOAD loci associated with $A \beta$ metabolism. Available data seem to be too far of these requisites, with the exception of $A P O E$, the most consistent across studies and across AD stages. Therefore, from our view, an unsuited sample size affecting statistical power or the use of the incorrect endophenotypes of $\mathrm{AD}$ could be preventing new discoveries.

\section{Brain genetic resistance factors: studies in healthy people}

The presence of Alzheimer-type pathology in healthy elderly people at death (Ref. 100) evidenced the existence of compensatory mechanisms avoiding a cognitive decline in populations. A GWAS developed in this group of subjects suggested the involvement of the RELN in the compensatory mechanism for $\mathrm{AD}$ (Ref. 101) and illustrated that studies on non-demented subjects with $\mathrm{AD}$ neuropathology are an interesting starting point to identify brain genetic resistance factors.

The state of resistance to brain insults, where the neuropathological hallmarks without clinical AD existing, has been defined as the cognitive reserve (CR). Individuals with higher CR tolerate the pathology for a longer duration and show signs of cognitive decline later in life (Ref. 102). Environmental and genetic factors are also believed to influence CR. It has been observed that the educational level (Ref. 103), work complexity (Ref. 104), engagement in leisure (Ref. 105), or social activity (Ref. 106) result in a reduced risk of dementia, contributing to CR. However, neuroplastic processes form the base of the above-mentioned factors. A study showed that the years of education is associated with genes involved in synaptic plasticity (Ref. 107), and not surprisingly, cognition and neuroplasticity seem to be driven by shared genes (Ref. 108).

The human cognition has a heritable component (Refs 109, 110). Cognition status is associated with several genetic variants, such as genes involved in oxidative stress (Ref. 111), biosynthesis of neurotransmitters (Ref. 112), ubiquitin metabolism and immune system (Ref. 113). It is to be noted that the immune system is highlighted as a prime pathway in LOAD
(Ref. 49) and is expected to be linked with cognition. The association of memory with $B D N F, 5 H T T$, and catechol-O-methyl transferase (COMT) genes remains more controversial (Ref. 114). However, as indicated, $B D N F$ and 5HTT genes have also been associated with the conversion of MCI to AD (Refs 72, 74). These make them suitable candidates for further studies in genetics with MCI or SCD subjects.

Recently, two studies conducted on non-demented elderly subjects have showed that genes related to $\mathrm{AD}$ (TOMM40, APOE, MEF $2 C$ and ABCGl) are significantly associated with the cognitive function (Refs 113, 115). This suggests that genes involved in the normal and pathological cognitions somehow overlap (Ref. 115) and highlight the applicability of the studies performed on healthy people. In addition, the cognition status has also been related to differential brain volumes (Ref. 116), thus, it seems that the HCV is a key component of the neuroanatomical basis of CR against memory in multiple sclerosis (Ref. 117). Thus, although the existence of a genetic component influencing cognition is evident, its relevance in the health and disease processes remains unclear. However, it cannot be denied that its knowledge can bring new insights.

Either way, the investigation of genetic variants affecting cognition and brain structure in healthy people with and without $\mathrm{AD}$ neuropathology could be a starting point to determine the intrinsic genetic resistance to dementia. The information obtained through these studies must be comprehensively translated to evaluate its clinical utility in the preclinical stages of $\mathrm{AD}$.

\section{Conclusion}

There exists an increasing interest in the characterization of the stages of pre-dementia. Taking into account the high genetic component of AD (Ref. 14), the identification of genetic variants influencing MCI and SCD can provide a new perspective in tackling the disease.

Recent technological improvements have promoted the identification of 28 genetic variants for LOAD. Despite that, data concerning pre-dementia stages remain scarce. At present, $A P O E$ gene is the most consistent association with risk to MCI and progression from MCI to AD (Ref. 54). The CLU locus has also showed promising results (Ref. 60). There are more inconsistent data for SCD. To the best of our knowledge, we are the first to show meta-analysed data evidencing the role of APOE as a risk factor for SCD (Fig. 2).

There is highly pronounced absence of genetic data for pre-dementia stage (Fig. 1). In addition, there is a high degree of heterogeneity between available studies in pre-dementia stage. In an extended way, the MCI studies show a statistical correlation between the genotype and neuropsychological test scores, which mainly provides informative data. In most 
cases, studies with SCD and MCI individuals have small sample sizes. Moreover, the consideration of population characteristics seems pertinent. SCD and MCI individuals comprise highly heterogeneous population, where converters to $\mathrm{AD}$ dementia coexist with converters to other forms of dementia and non-converters. In that scenario, the identification of novel and expected LOAD loci may be hampered by the effect size of the true $\mathrm{AD}$ group. That could explain the reduction in the effect size of $A P O E$ along stages. On the other hand, the identification of progression factors that are not previously reported must be considered with prudence until their validation. This limitation points to the necessity of using larger cohorts in studies involving a pre-dementia stage.

Efforts are required to provide useful data, which can help in designing strategies to stop or modulate the course of the disease. In that sense, a GWAS in the MCI population seems mandatory. Moreover, the identification of the genetic factors conferring resilience to dementia in non-demented people could provide a good opportunity in uncovering the compensatory mechanisms that may prevent the disease progression. Therefore, a genome-wide approach for endophenotypes involved in CR also seems affordable and advisable.

In conclusion, genetic research in the pre-dementia stages of non-demented people must be potentiated to obtain advances in $\mathrm{AD}$ and design prevention strategies.

\section{Acknowledgements}

We are indebted to Trinitat Port-Carbó and her family for their support to the Fundació ACE research programs. Fundació ACE collaborates with the Centro de Investigación Biomédica en Red sobre Enfermedades Neurodegenerativas (CIBERNED, Spain) and is one of the participating centers of the Dementia Genetics Spanish Consortium (DEGESCO). The present work has been performed as part of the Biochemistry, Molecular Biology and Biomedicine doctoral program of S. Moreno-Grau at Universitat Autònoma de Barcelona (Barcelona, Spain).

\section{Financial support}

This work is supported by Grant PI13/02434 (Acción Estratégica en Salud. Instituto de Salud Carlos III (ISCIII). Ministerio de Economía y Competitividad, Spain), and Obra Social 'La Caixa' (Barcelona, Spain).

\section{Conflict of interest}

None.

\section{References}

1. Sperling R.A. et al. (2011) Toward defining the preclinical stages of Alzheimer's disease: recommendations from the National Institute on Aging-Alzheimer's Association workgroups on diagnostic guidelines for Alzheimer's disease. Alzheimer's and Dementia 7, 280-292

2. Albert M.S et al. (2011) The diagnosis of mild cognitive impairment due to Alzheimer's disease: recommendations from the National Institute on Aging-Alzheimer's
Association workgroups on diagnostic guidelines for Alzheimer's disease. Alzheimer's and Dementia 7, 270-279

3. McKhann G.M. et al. (2011) The diagnosis of dementia due to Alzheimer's disease: recommendations from the National Institute on Aging-Alzheimer's Association workgroups on diagnostic guidelines for Alzheimer's disease. Alzheimer's and Dementia 7, 263-269

4. Caselli R.J. et al. (2009) Longitudinal modeling of age-related memory decline and the APOE epsilon4 effect. New England Journal of Medicine 361, 255-263

5. Jessen F. et al. (2014) A conceptual framework for research on subjective cognitive decline in preclinical Alzheimer's disease. Alzheimer's and Dementia 10, 844-852

6. Reisberg B. et al. (2010) Outcome over seven years of healthy adults with and without subjective cognitive impairment. Alzheimer's and Dementia 6, 11-24

7. Amieva H. (2008) Prodromal Alzheimer's disease: successive emergence of the clinical symptoms. Annals of Neurology 64, 492-498

8. Jonker C., Geerlings M.I. and Schmand B. (2000) Are memory complaints predictive for dementia? A review of clinical and population-based studies. International Journal of Geriatrics Psychiatry 15, 983-991

9. Jessen F. (2010) Prediction of dementia by subjective memory impairment. Archive of General Psychiatry 67, 414-422

10. Petersen R.C. et al. (1999) Mild cognitive impairment: clinical characterization and outcome. Archives of Neurology 56, 303-308

11. Espinosa A. et al. (2013) A longitudinal follow-up of 550 mild cognitive impairment patients: evidence for large conversion to dementia rates and detection of major risk factors involved. Journal of Alzheimer's Disease 34, 769-780

12. Jack C.R. et al. (2010) Hypothetical model of dynamic biomarkers of the Alzheimer's pathological cascade. Lancet Neurology 9, 119-128

13. Drago V. et al. (2011) Disease tracking markers for Alzheimer's disease at the prodromal (MCI) stage. Journal of Alzheimer's Disease 26 (Suppl 3), 159-199

14. Avramopoulos D. (2009) Genetics of Alzheimer's disease: recent advances. Genome Medicine 1, 34

15. Goldman J.S. et al. (2011) Genetic counseling and testing for Alzheimer disease: joint practice guidelines of the American College of Medical Genetics and the National Society of Genetic Counselors. Genetics in Medicine: Official Journal of the American College of Medical Genetics 13, 597-605

16. Goate A. et al. (1991) Segregation of a missense mutation in the amyloid precursor protein gene with familial Alzheimer's disease. Nature 349, 704-706

17. Sherrington R. et al. (1995) Cloning of a gene bearing missense mutations in early-onset familial Alzheimer's disease. Nature 375, 754-760

18. Levy-Lahad E., Wijsman E.M. and Nemens E. (1995) A familial Alzheimer's disease locus on chromosome 1. Science 411, 16-19

19. Raux G. et al. (2005) Molecular diagnosis of autosomal dominant early onset Alzheimer's disease: an update. Journal of Medical Genetics 42, 793-795

20. Corder E and Saunders A (1993) Gene dose of apolipoprotein E type 4 allele and the risk of Alzheimer's disease in late onset families. Science 261, 921-923

21. Genin E. et al. (2011) APOE and Alzheimer disease: a major gene with semi-dominant inheritance. Molecular Psychiatry 16, 903-907

22. Rogaeva E. et al. (2007) The neuronal sortilin-related receptor SORL1 is genetically associated with Alzheimer disease. Nature Genetics 39, 168-177

23. Lambert J.C. et al. (2013) Meta-analysis of 74,046 individuals identifies 11 new susceptibility loci for Alzheimer's disease. Nature Genetics 45, 1452-1458

24. Harold D. et al. (2009) Genome-wide association study identifies variants at CLU and PICALM associated with Alzheimer's disease. Nature Genetics 41, 1088-1093

25. Seshadri S. et al. (2011) Genome-wide analysis of genetic loci associated with Alzheimer's disease. JAMA 303, 1832-1840

26. Hollingworth P. et al. (2011) Common variants at ABCA7, MS4A6A/MS4A4E, EPHA1, CD33 and CD2AP are associated with Alzheimer's disease. Nature Genetics 43, 429-435

27. Jun G. et al. (2010) Meta-analysis confirms CR1, CLU, and PICALM as Alzheimer disease risk loci and reveals 
interactions with APOE genotypes. Archives of Neurology 67, 1473-1484

28. Carrasquillo M. et al. (2011) Replication of BIN1 association with Alzheimer's disease and evaluation of genetic interactions. Journal of Alzheimer's Disease 24, 751-758

29. Lee J.H. et al. (2011) Identification of novel loci for Alzheimer disease and replication of CLU, PICALM, and BIN1 in Caribbean Hispanic individuals. Archives of Neurology 68, 320-328

30. Yu J-T. (2013) Genetic variation in clusterin gene and Alzheimer's disease risk in Han Chinese. Neurobiology of Aging 34, 1921.e17-1921.e23

31. Jin C. et al. (2012) Association of the CR1 polymorphism with late-onset Alzheimer's disease in Chinese Han populations: a meta-analysis. Neuroscience Letters 527, 46-49

32. Carrasquillo M.M. et al. (2011) Replication of EPHA1 and CD33 associations with late-onset Alzheimer's disease: a multi-centre case-control study. Molecular Neurodegeneration 6, 54

33. Reitz C. et al. (2013) Variants in the ATP-binding cassette transporter (ABCA7), apolipoprotein $\mathrm{E} \in 4$, and the risk of late-onset Alzheimer disease in African Americans. JAMA 309, 1483-1492

34. Ruiz A. et al. (2014) Follow-up of loci from the International Genomics of Alzheimer's disease project identifies TRIP4 as a novel susceptibility gene. Translational Psychiatry 4, e358

35. Jun G. et al. (2016) A novel Alzheimer disease locus located near the gene encoding tau protein. Molecular Psychiatry 21, 108-117

36. Escott-Price V. (2014) Gene-wide analysis detects two new susceptibility genes for Alzheimer's disease. PLOS ONE 9, e94661

37. Boada M. et al. (2014) ATP5H/KCTD2 locus is associated with Alzheimer's disease risk. Molecular Psychiatry 19, 682-687

38. Jonsson T. et al. (2012) A mutation in APP protects against Alzheimer's disease and age-related cognitive decline. Nature 488, 96-99

39. Guerreiro R. et al. (2013) TREM2 variants in Alzheimer's disease. New England Journal of Medicine 368, 117-127

40. Cruchaga C. et al. (2014) Rare coding variants in the phospholipase D3 gene confer risk for Alzheimer's disease. Nature 505, 550-554

41. Wetzel-Smith M.K. et al. (2014) A rare mutation in UNC5C predisposes to late-onset Alzheimer's disease and increases neuronal cell death. Nature Medicine 20, 1452-1457

42. Benitez B.A. et al. (2013) TREM2 is associated with the risk of Alzheimer's disease in Spanish population. Neurobiology of Aging 34, 1711.e15-1711.e17

43. Ruiz A. et al. (2014) Assessing the role of the TREM2 p.R47H variant as a risk factor for Alzheimer's disease and frontotemporal dementia. Neurobiology of Aging 35, 444.e1444.e4

44. Paloneva J. (2002) Mutations in two genes encoding different subunits of a receptor signaling complex result in an identical disease phenotype. American Journal of Human Genetics 71, 656-662

45. Thelen M. (2014) Investigation of the role of rare TREM2 variants in frontotemporal dementia subtypes. Neurobiology of Aging 35, 2657.e13-2657.e19

46. Hooli B.V. et al. (2015) PLD3 gene variants and Alzheimer's disease. Nature 520, E7-E8

47. Heilmann S. et al. (2015) PLD3 in non-familial Alzheimer's disease. Nature 520, E3-E5

48. Lambert J-C. et al. (2010) Implication of the immune system in Alzheimer's disease: evidence from genome-wide pathway analysis. Journal of Alzheimer's Disease 20, 1107-1118

49. International Genomics of Alzheimer's disease Consortium (IGAP) (2014) Convergent genetic and expression data implicate immunity in Alzheimer's disease. Alzheimer's and Dementia 14, 02492-02493

50. Petersen R.C. et al. (1995) Apolipoprotein E status as a predictor of the development of Alzheimer's disease in memory-impaired individuals. JAMA 273, 1274-1278

51. Fleisher A.S. et al. (2007) Clinical predictors of progression to Alzheimer disease in amnestic mild cognitive impairment. Neurology 68, 1588-1595
52. Artero S. et al. (2008) Risk profiles for mild cognitive impairment and progression to dementia are gender specific. Journal of Neurology Neurosurgery and Psychiatry 79, 979-984

53. Fei M. and Jianhua W. (2013) Apolipoprotein $\varepsilon 4$-allele as a significant risk factor for conversion from mild cognitive impairment to Alzheimer's disease: a meta-analysis of prospective studies. Journal of Molecular Neuroscience 50, $257-263$

54. Elias-Sonnenschein L.S. et al. (2011) Predictive value of APOE- $\varepsilon 4$ allele for progression from MCI to AD-type dementia: a meta-analysis. Journal of Neurology Neurosurgery and Psychiatry 82, 1149-1156

55. Lopez O.L. et al. (2003) Risk factors for mild cognitive impairment in the Cardiovascular Health Study Cognition Study: part 2. Archives of Neurology 60, 1394-1399

56. Corder E.H. et al. (1994) Protective effect of apolipoprotein $\mathrm{E}$ type 2 allele for late onset Alzheimer disease. Nature Genetics 7, $180-184$

57. Wilson R.S. et al. (2002) The apolipoprotein E epsilon 2 allele and decline in episodic memory. Journal of Neurology Neurosurgery and Psychiatry 73, 672-677

58. Blacker D. (2007) Neuropsychological measures in normal individuals that predict subsequent cognitive decline. Archives of Neurology 64, 862-871

59. Bonner-Jackson A., Okonkwo O. and Tremont G. (2012) Apolipoprotein E $\varepsilon 2$ and functional decline in amnestic mild cognitive impairment and Alzheimer disease. American Journal of Geriatrics Psychiatry 20, 584-593

60. Lacour A. et al. (2016) Genome-wide significant risk factors for Alzheimer's disease: role in progression to dementia due to Alzheimer's disease among subjects with mild cognitive impairment. Molecular Psychiatry advance online publication. doi: $10.1038 / \mathrm{mp} .2016 .18$.

61. Chibnik L.B. et al. (2011) CR1 is associated with amyloid plaque burden and age-related cognitive decline. Annals of Neurology 69, 560-569

62. Carrasquillo M.M. et al. (2015) Late-onset Alzheimer's risk variants in memory decline, incident mild cognitive impairment, and Alzheimer's disease. Neurobiology of Aging 36, 60-67

63. Hu X. et al. (2011) Genome-wide association study identifies multiple novel loci associated with disease progression in subjects with mild cognitive impairment. Translational Psychiatry 1, e54

64. Thambisetty M. et al. (2013) Alzheimer risk variant CLU and brain function during aging. Biological Psychiatry 73, 399-405

65. Rodríguez-Rodríguez E. et al. (2013) Genetic risk score predicting accelerated progression from mild cognitive impairment to Alzheimer's disease. Journal of Neuronal Transmission 120, 807-812

66. Adams H. et al. (2015) Genetic risk of neurodegenerative disease is associated with mild cognitive impairment and conversion to dementia. Alzheimer's and Dementia 15, 118-111

67. Samaranch L. et al. (2010) The effect of MAPT H1 and APOE $\varepsilon 4$ on transition from mild cognitive impairment to dementia. Journal of Alzheimer's Disease 22, 1065-1071

68. Di Maria E. et al. (2010) The H1 haplotype of the tau gene (MAPT) is associated with mild cognitive impairment. Journal of Alzheimer's Disease 19, 909-914

69. Cruchaga C. et al. (2010) SNPs associated with cerebrospinal fluid phospho-tau levels influence rate of decline in Alzheimer's disease. PLoS Genetics 6, e1001101

70. Myers A.J. et al. (2005) The H1c haplotype at the MAPT locus is associated with Alzheimer's disease. Human Molecular Genetics 14, 2399-2404

71. Chiappelli M. et al. (2006) VEGF gene and phenotype relation with Alzheimer's disease and mild cognitive impairment. Rejuvenation Research 9, 485-493

72. Forlenza O.V. et al. (2010) Effect of brain-derived neurotrophic factor Val66Met polymorphism and serum levels on the progression of mild cognitive impairment. World Journal Biological Psychiatry 11, 774-780

73. Ferris S. et al. (2009) Progression from mild cognitive impairment to Alzheimer's disease: effects of sex, butyrylcholinesterase genotype, and rivastigmine treatment. Pharmacogenetics and Genomics 19, 635-646 
74. Marini S. et al. (2011) Implication of serotonin-transporter (5-HTT) gene polymorphism in subjective memory complaints and mild cognitive impairment (MCI). Archives of Gerontology and Geriatrics 52, e71-e74

75. Strange B.A. et al. (2008) Emotion-induced retrograde amnesia is determined by a 5-HTT genetic polymorphism. Journal of Neuroscience 28, 7036-7039

76. Koutroumani M. et al. (2013) The deletion variant of $a 2 b-$ adrenergic receptor is associated with decreased risk in Alzheimer's disease and mild cognitive impairment. Journal of Neurological Sciences 328, 19-23

77. Zill P. et al. (2000) Polymorphisms in the alpha-2 macroglobulin gene in psychogeriatric patients. Neuroscience Letters 294, 69-72

78. Laws S.M. et al. (2002) Association between the presenilin-1 mutation Glu318Gly and complaints of memory impairment. Neurobiology of Aging 23, 55-58

79. Watfa G. et al. (2010) Association study of gene polymorphisms involved in vascular alterations in elderly hypertensives with subjective memory complaints. Dementia Geriatrics Cognitive Disorders 30, 440-448

80. Lau S. et al. (2012) Functional effects of genetic polymorphism in inflammatory genes in subjective memory complainers. Neurobiology of Aging 33, 1054-1056

81. Fleisher A.S. et al. (2013) Apolipoprotein E $\varepsilon 4$ and age effects on florbetapir positron emission tomography in healthy aging and Alzheimer disease. Neurobiology of Aging 34, 1-12

82. Risacher S.L. et al. (2015) APOE effect on Alzheimer's disease biomarkers in older adults with significant memory concern. Alzheimers and Dementia 11, 1417-1429

83. Mormino E.C. et al. (2014) Amyloid and APOE $\varepsilon 4$ interact to influence short-term decline in preclinical Alzheimer disease. Neurology 82, 1760-1767

84. Ramanan V.K. et al. (2014) APOE and BCHE as modulators of cerebral amyloid deposition: a florbetapir PET genomewide association study. Molecular Psychiatry 19, 351-357

85. Replogle J. et al. (2015) A TREM1 variant alters the accumulation of Alzheimer-related amyloid pathology. Annals of Neurology 77, 469-477

86. Ramanan V.K. et al. (2015) GWAS of longitudinal amyloid accumulation on 18F-florbetapir PET in Alzheimer's disease implicates microglial activation gene IL1RAP. Brain 138, 3076-3088

87. Louwersheimer E. et al. (2015) The influence of genetic variants in SORL1 gene on the manifestation of Alzheimer's disease. Neurobiology of Aging 36, 1605.e13-1605.e20

88. Lancaster T.M. et al. (2015) Alzheimer's disease risk variant in CLU is associated with neural inefficiency in healthy individuals. Alzheimers and Dementia 11, 1144-1152

89. Chauhan G. et al. (2015) Association of Alzheimer's disease GWAS loci with MRI markers of brain aging. Neurobiology of Aging 36, 1765.e7-1765.16

90. Hibar D.P. et al. (2015) Common genetic variants influence human subcortical brain structures. Nature 520, 224-229

91. Meda S. et al. (2013) Genetic interactions associated with 12month atrophy in hippocampus and entorhinal cortex in Alzheimer's Disease Neuroimaging Initiative. Neurobiology of Aging 34, 1518.e9-1518.18

92. Sabuncu M. (2011) The association between a polygenic Alzheimer score and cortical thickness in clinically normal subjects. Cerebral Cortex 22, 2653-2661

93. Elias-Sonneschein L. et al. (2013) Genetic loci associated with Alzheimer's disease and cerebrospinal fluid biomarkers in a Finnish case-control cohort. PLOS ONE 8, e59676

94. Cruchaga C. et al. (2013) GWAS of cerebrospinal fluid tau levels identifies risk variants for Alzheimer's disease. Neuron 78, 256-268

95. Ramirez A. et al. (2014) SUCLG2 identified as both a determinator of CSF A $\beta 1-42$ levels and an attenuator of cognitive decline in Alzheimer's disease. Human Molecular Genetics 23, 6644-6658

96. Kauwe J. et al. (2011) Fine mapping of genetic variants in BIN1, CLU, CR1 and PICALM for association with cerebrospinal fluid biomarkers for Alzheimer's disease. PLoS ONE 6, e15918

97. Martinskainen H. et al. (2015) Effects of Alzheimer's diseaseassociated risk loci on cerebrospinal fluid biomarkers and disease progression: a polygenic risk score approach. Journal of Alzheimer's Disease 43, 565-573

98. Cruchaga C., Ebbert M., Kauwe J. (2014) Genetic discoveries in AD using CSF amyloid and tau. Current Genetic Medicine Reports 2, 23-29

99. Fagan A.M. et al. (2006) Inverse correlation between in vivo amyloid imaging load and cerebrospinal fluid Abeta42 in humans. Annals of Neurology 59, 512-519

100. Neuropathology Group. Medical Research Council Cognitive Function and Aging Study (2001) Pathological correlates of late-onset dementia in a multicentre, community-based population in England and Wales. Neuropathology Group of the Medical Research Council Cognitive Function and Ageing Study (MRC CFAS). Lancet 357, 169-175

101. Kramer P.L. et al. (2011) Alzheimer disease pathology in cognitively healthy elderly: a genome-wide study. Neurobiology of Aging 32, 2113-2122

102. Stern Y. (2012) Cognitive reserve in ageing and Alzheimer's disease. Lancet Neurology 11, 1006-1012

103. Sando S.B. et al. (2008) Risk-reducing effect of education in Alzheimer's disease. International Journal of Geriatric Psychiatry 23, 1156-1162

104. Andel R. et al. (2005) Complexity of work and risk of Alzheimer's disease: a population-based study of Swedish twins. Journals of Gerontology: Series B 60, P251-P258

105. Scarmeas N. et al. (2001) Influence of leisure activity on the incidence of Alzheimer's disease. Neurology 57, 2236-2242

106. Wilson R.S. (2009) Elderly women with larger social networks are less likely to develop dementia. Evidence-Based Mental Health 12, 22

107. Rietveld C. et al. (2014) Common genetic variants associated with cognitive performance identified using the proxyphenotype method. Proceedings of the National Academy of Sciences of the United States of America 111, 13790-13794

108. Van Veluw S.J. et al. (2012) Prefrontal cortex cytoarchitecture in normal aging and Alzheimer's disease: a relationship with IQ. Brain structure and function 217, 797-808

109. Deary I.J., Johnson W. and Houlihan L.M. (2009) Genetic foundations of human intelligence. Human Genetics 126, 215-232

110. Glahn D.C. (2013) Genetic basis of neurocognitive decline and reduced white-matter integrity in normal human brain aging. Proceedings of the National Academy of Sciences of the United States of America 110, 19006-19011

111. Harris S.E. et al. (2007) A genetic association analysis of cognitive ability and cognitive ageing using 325 markers for 109 genes associated with oxidative stress or cognition. $B M C$ Genetics 8, 43

112. Barral S. et al. (2014) Common genetic variants on 6q24 associated with exceptional episodic memory performance in the elderly. Journal of the American Medical Association Neurology 71, 1514-1519

113. Debette S. et al. (2014) Genome-wide studies of verbal declarative memory in non-demented older people: the CHARGE consortium. Biological Psychiatry 8, 749-773

114. Sabb F.W. et al. (2009) Challenges in phenotype definition in the whole-genome era: multivariate models of memory and intelligence. Neuroscience 164, 88-107

115. Davies G. et al. (2015) Genetic contributions to variation in general cognitive function: a meta-analysis of genome-wide association studies in the CHARGE consortium $(\mathrm{N}=53$ 949). Molecular Psychiatry 20, 183-192

116. Dowling N.M. et al. (2011) Neuropathological associates of multiple cognitive functions in two community-based cohorts of older adults. Journal of the International Neuropsychological Society 17, 602-614

117. Sumowski J.F. et al. (2015) Searching for the neural basis of reserve against memory decline: intellectual enrichment linked to larger hippocampal volume in multiple sclerosis. European Journal of Neurology 23, 39-44

118. Bertram L. et al. (2007) Systematic meta-analyses of Alzheimer disease genetic association studies: the Alzgene database. Nature Genetics 39, 17-23

119. Lambert J. (2009) Genome-wide association study identifies variants at CLU and CR1 associated with Alzheimer's disease. Nature Genetics 41, 1094-1099

120. Krzywinski M. et al. (2009) Circos: an information aesthetic for comparative genomics. Genome Research 19, 1639-1645 
121. Wang X. et al. (2014) Frequency of the apolipoprotein E $\varepsilon 4$ allele in a memory clinic cohort in Beijing: a naturalistic descriptive study. PLos ONE 9, e99130

122. Jin C. et al. (2014) The SORL1 polymorphism rs985421 may confer the risk for amnestic mild cognitive impairment and Alzheimer's disease in the Han Chinese population. Neuroscience Letter 563, 80-81

123. Sasaki M. et al. (2009) Prevalence of four subtypes of mild cognitive impairment and APOE in a Japanese community. International Journal of Geriatric Psychiatry 24, 11191126

124. Kim S.M. et al. (2013) Regional cerebral perfusion in patients with Alzheimer's disease and mild cognitive impairment: effect of APOE Epsilon4 allele. Neuroradiology 55, 25-34

125. Foster J.K. et al. (2013) Lack of reliable evidence for a distinctive $\varepsilon 4$-related cognitive phenotype that is independent from clinical diagnostic status: findings from the Australian Imaging, Biomarkers and Lifestyle Study. Brain 136, 22012216

126. Troyer A.K. et al. (2012) Associative recognition in mild cognitive impairment: relationship to hippocampal volume and apolipoprotein E. Neuropsychologia 50, 3721-3728

127. Traykov L et al. (1999) Apolipoprotein E phenotypes in demented and cognitively impaired patients with and without cerebrovascular disease. European Journal of Neurology 6, 415-421

128. Luck T. et al. (2010) Risk factors for incident mild cognitive impairment-results from the German Study on Ageing, Cognition and Dementia in Primary Care Patients (AgeCoDe). Acta Psychiatrica Scandinavica 121, 260-272

129. Chico L. et al. (2013) Oxidative stress and APOE polymorphisms in Alzheimer's disease and in mild cognitive impairment. Free Radical Research 47, 569-576

130. Siuda J. et al. (2009) From mild cognitive impairment to Alzheimer's disease - influence of homocysteine, vitamin B12 and folate on cognition over time: results from one-year follow-up. Neurologia i neurogirurgia Polska 43, 321-329

131. Cervantes S et al. (2011) Genetic variation in APOE cluster region and Alzheimer's disease risk. Neurobiology of Aging 32, 2107.e7-2107.17

132. Bartrés-Faz D. et al. (2001) Neuropsychological and genetic differences between age-associated memory impairment and mild cognitive impairment entities. Journal of the American Geriatrics Society 49, 985-990

133. Buerger K. et al. (2005) Increased levels of CSF phosphorylated tau in apolipoprotein E epsilon4 carriers with mild cognitive impairment. Neuroscience Letter 391, 48-50
134. Zekry D. et al. (2010) Telomere length and ApoE polymorphism in mild cognitive impairment, degenerative and vascular dementia. Journal of Neurological Science 299, 108-111

135. Jefferson A.L. et al. (2014) APOE and mild cognitive impairment: the Framingham Heart Study. Age and Ageing 2, 307-311

136. Altmann A. et al. (2014) Sex modifies the APOE-related risk of developing Alzheimer disease. Annals of Neurology 75, 563-573

137. Tyas S.L. et al. (2007) Transitions to mild cognitive impairments, dementia, and death: findings from the Nun Study. American Journal of Epidemiology 165, 1231-1238

138. O'Bryant S.E. et al. (2013) Risk factors for mild cognitive impairment among Mexican Americans. Alzheimers and Dementia 9, 622-631

139. Boyle P.A. et al. (2010) The APOE \&4 Allele is associated with incident mild cognitive impairment among communitydwelling older persons. Neuroepidemiology 34, 43-49

140. Stewart R. et al. (2001) Depression, APOE genotype and subjective memory impairment: a cross-sectional study in an African-Caribbean population. Psychological Medicine 31, 431-440

141. Laws S.M. et al. (2002) APOE-epsilon4 and APOE -491A polymorphisms in individuals with subjective memory loss. Molecular Psychiatry 7, 768-775

142. Verdile G. et al. (2012) Associations between gonadotropins, testosterone and $\beta$ amyloid in men at risk of Alzheimer's disease. Molecular Psychiatry 19, 69-75

143. Jessen F. et al. (2007) Patterns of subjective memory impairment in the elderly: association with memory performance. Psychological Medicine 37, 1753-1762

144. Risacher S.L. et al. (2015) APOE effect on Alzheimer's disease biomarkers in older adults with significant memory concern. Alzheimers and Dementia 11, 1417-1429

145. Caselli R.J. et al. (2014) Subjective cognitive decline: self and informant comparisons. Alzheimers and Dementia 10, 93-98

146. Samieri C. et al. (2014) Subjective cognitive concerns, episodic memory, and the APOE $\varepsilon 4$ allele. Alzheimer's and Dementia 10, 752-759

*Corresponding author:

Agustín Ruiz,

Fundació ACE

Barcelona Alzheimer Treatment and Research Centre,

C/Marquès de Sentmenat, 57, 08029 Barcelona, Spain.

Tel: +3493.444.73.18;

Fax: +3493.410 .17 .01$

E-mail: aruiz@fudacioace.com 